\title{
Clozapine Alone versus Clozapine and Risperidone with Refractory Schizophrenia
}

\author{
William G. Honer, M.D., Allen E. Thornton, Ph.D., Eric Y.H. Chen, M.D., \\ Raymond C.K. Chan, Ph.D., Jessica O.Y. Wong, M.B., Andrea Bergmann, M.D., \\ Peter Falkai, M.D., Edith Pomarol-Clotet, M.D., Peter J. McKenna, M.B., \\ Emmanuel Stip, M.D., Richard Williams, M.B., G. William MacEwan, M.D., \\ Kishor Wasan, Ph.D., and Ric Procyshyn, Ph.D., \\ for the Clozapine and Risperidone Enhancement (CARE) Study Group*
}

ABSTRACT

From the Department of Psychiatry (W.G.H., R.W., G.W.M.) and the Division of Pharmaceutics and Biopharmaceutics (K.W.), University of British Columbia Vancouver, Canada; Department of Psychology, Simon Fraser University, Burnaby, B.C., Canada (A.E.T.); Department of Psychiatry, University of Hong Kong, Hong Kong, China (E.Y.H.C.); Department of Psychology, Sun Yat-Sen University, Guang zhou, China (R.C.K.C.); Castle Peak Hospital, Hong Kong, China (J.O.Y.W.) Department of Psychiatry, Saarland University, Homburg, Germany (A.B., P.F.) Department of Psychiatry, University of Cambridge, Cambridge, United Kingdom (E.P.-C., P.J.M.); Department of Psychiatry, University of Montreal, Montreal (E.S.) and Riverview Hospital, Port Coquitlam B.C., Canada (R.P.). Address reprint requests to Dr. Honer at the Centre for Complex Disorders and the Department of Psychiatry, University of British Columbia, Vancouver General Hospital Research Pavilion, 828 W. 10th Ave., Suite 211, Vancouver, BC V5Z 1L8, Canada, or athoner@interchange.ubc.ca.

*The members of the CARE Study Group are listed in the Appendix.

N Engl J Med 2006;354:472-82.

Copyright $(2006$ Massachusetts Medical Society.
BACKGROUND

The treatment of schizophrenia with multiple antipsychotic drugs is common, but the benefits and risks are not known.

\section{METHODS}

In a randomized, double-blind study, we evaluated patients with schizophrenia and a poor response to treatment with clozapine. The patients continued to take clozapine and were randomly assigned to receive eight weeks of daily augmentation with $3 \mathrm{mg}$ of risperidone or with placebo. This course of treatment was followed by an optional 18 weeks of augmentation with risperidone. The primary outcome was reduction in the total score for severity of symptoms on the Positive and Negative Syndrome Scale (PANSS). The secondary outcomes included cognitive functioning.

\section{RESULTS}

A total of 68 patients were randomly assigned to treatment. In the double-blind phase, the mean total score for the severity of symptoms decreased from baseline to eight weeks in both the risperidone and the placebo groups. There was no statistically significant difference in symptomatic benefit between augmentation with risperidone and placebo: 9 of 34 patients receiving placebo and 6 of 34 receiving risperidone responded to treatment $(\mathrm{P}=0.38)$. The mean difference in the change in PANSS scores from baseline to eight weeks between those receiving risperidone and those receiving placebo was 0.1 (95 percent confidence interval, -7.3 to 7.0). The verbal working-memory index showed a small decline in the risperidone group and a small improvement in the placebo group $(\mathrm{P}=0.02$ for the comparison between the two groups in the change from baseline). The increase in fasting blood glucose levels was mildly greater in the risperidone group than in the placebo group (16.2 vs. $1.8 \mathrm{mg}$ per deciliter [0.90 vs. $0.10 \mathrm{mmol}$ per liter], $\mathrm{P}=0.04$ ). The incidence and severity of other side effects did not differ between the two groups.

CONCLUSIONS

In this short-term study, the addition of risperidone to clozapine did not improve symptoms in patients with severe schizophrenia. (ClinicalTrials.gov number, NCT00272584) 
E VEN WITH ADEQUATE TREATMENT, AS little as 20 percent of patients with schizophrenia have a complete resolution of symptoms, whereas up to one third have a clinically inadequate response. ${ }^{1}$ Poor response of psychotic symptoms to single antipsychotic drugs has been cited as the most common reason for simultaneous prescription of multiple antipsychotic drugs, or polypharmacy. ${ }^{2,3}$ Antipsychotic polypharmacy is increasingly common, although the evidence of its efficacy from randomized, controlled trials is limited or contradictory, there is a risk of exacerbation of side effects, and the cost implications are substantial. ${ }^{4-14}$ If antipsychotic polypharmacy is of value, treatment with combinations of drugs with complementary receptor-binding properties might have the highest likelihood of success. ${ }^{15}$ Clozapine does not saturate dopamine D2 receptors, even when given at high doses, whereas risperidone occupies a high proportion of dopamine D2 receptors when given at clinically used doses. ${ }^{16}$ Combining drugs that have complementary profiles of cognitive improvement might be of further benefit. Clozapine and risperidone both appear to improve frontal-lobe function, although risperidone may have more benefit for verbal working memory. ${ }^{17}$

We investigated whether augmentation with risperidone would alleviate psychotic symptoms in patients with an incomplete response to treatment with clozapine at the usual clinical dose over an appropriate period of time. The secondary hypotheses were that risperidone augmentation would improve cognitive functioning and that the side effects of clozapine plus risperidone would be similar to those of clozapine alone.

METHODS

\section{PARTICIPANTS}

We enrolled 71 patients from seven institutions in Canada, Germany, China, and the United Kingdom that were similar in the systems and quality of care to publicly funded, academic-affiliated medical centers in the United States. Both inpatients and outpatients were eligible. The inclusion criteria were a diagnosis of schizophrenia or schizoaffective disorder according to the criteria of the American Psychiatric Association's Diagnostic and Statistical Manual of Mental Disorders, fourth edition (DSM-IV) ${ }^{18}$; age from 18 through 65 years; treatment with clozapine (Clozaril, Novartis) for the indication of poor response to other antipsychotic agents; treatment for at least 12 weeks at a stable dose of $400 \mathrm{mg}$ or more per day, unless the size of the dose was limited by side effects; a total score of 80 or greater at baseline on the Positive and Negative Syndrome Scale (PANSS) ${ }^{19}$ (the range of possible scores is 30 to 210 , with higher scores indicating more severe symptoms); a Clinical Global Impressions (CGI) ${ }^{20}$ score of 4 or greater (range of possible scores, 1 [not mentally ill] to 7 [extremely ill]); and a Social and Occupational Functioning Assessment Scale (SOFAS) ${ }^{18}$ score of 40 or less (range of possible scores, 1 to 100, with lower scores indicating impaired functioning). The exclusion criteria were clinically significant alcohol or substance abuse in the previous three months, developmental disability, current treatment with clozapine for the primary indication of movement disorder or of intolerable side effects from other medications, or previous treatment with clozapine augmented with risperidone. Patients were required to discontinue any antipsychotic drugs other than clozapine, any mood-stabilizing or antidepressant drugs, and any anticonvulsant drugs for at least two weeks before entry into the study (except for fluoxetine and electroconvulsive therapy, which were discontinued for at least four weeks before entry into the study). Concomitant medications for stable medical conditions were permitted.

\section{STUDY DESIGN}

From June 2001 through January 2004, patients were enrolled and entered a one-week phase of single-blind placebo augmentation. On day 7, patients with an improvement in the overall PANSS score of 20 percent or greater were withdrawn from the study; all others were randomly assigned to double-blind treatment with risperidone or a placebo of identical appearance. Randomization was performed according to a computer-generated schedule with a permuted-block design. The fixed block size was four patients. The site investigators did not know the block size. The person generating the randomization schedule was not involved in determining patients' eligibility, administering treatment, or determining outcome. The patients were assigned in sequence at each site. Throughout the study, the patients, site investigators, and raters remained blinded.

Risperidone (Risperdal, Janssen Pharmaceutica) was administered as 1-mg tablets; the dose was 
increased to $3 \mathrm{mg}$ per day over the first 15 days. The investigators were allowed to decrease the dose by one tablet per day if the side effects were intolerable. Adherence to treatment with the study drugs was monitored by pill counts of the medications, which were provided in numbered blister packs. The serum levels of clozapine and its metabolite norclozapine were determined on days 7 , 63, and 189; risperidone levels were not measured. Lorazepam (at a maximum dose of $6 \mathrm{mg}$ per day) or chloral hydrate (maximum dose, $2 \mathrm{~g}$ per day) was permitted for treatment of agitation or other symptoms. No lorazepam or chloral hydrate was permitted for 48 hours before cognitive testing. Anticholinergic drugs were allowed only for the treatment of acute side effects. After the doubleblind phase, the patients were offered the option of receiving unblinded augmentation of clozapine treatment with risperidone for an additional 18 weeks.

Janssen-Ortho, Canada, provided the risperidone and matching placebo and reviewed the protocol; there was no request for amendment. The only study data provided to Janssen-Ortho consisted of reports of serious adverse events. The data were analyzed and the manuscript was written solely by the listed authors. Ethics approval was obtained from the institutional review board at each site. Written informed consent was obtained from all patients.

\section{BASELINE ASSESSMENT AND EFFICACY MEASURES}

The primary outcome measure was severity of symptoms, measured by the total PANSS score on days 7, 35, and 63. The PANSS is a widely used instrument in clinical trials of schizophrenia treatment, with well-described psychometric properties, including reliability. ${ }^{19}$ The raters, all of whom were trained in administration of the PANSS, reviewed standardized videotaped interviews. The raters were fluent in English, and each center was led by an academic research psychiatrist. The secondary measures were the PANSS positive and negative symptom scores and the CGI severity scores. Patients with a 20 percent or greater reduction in total PANSS score were classified as having a response.

Frontal-lobe cognitive function, an additional secondary outcome measure, was assessed on days 7, 63, and 189. Testing was carried out under the supervision of neuropsychologists. Working-memory function was operationally defined as the limited-capacity, short-term facility for the simultaneous storage and processing of information. Two measures of verbal working memory were employed, the Brown-Peterson procedure $^{21,22}$ and the Letter-Number Sequencing (LNS) task. ${ }^{23}$ Both tasks require the temporary storage of information and either simultaneous backward counting (Brown-Peterson) or mental manipulation of the material (LNS). An aggregate composite index of verbal working memory was formed by combining standardized $z$ scores of the tasks from each time point. The baseline means and standard deviations for the two working-memory tasks were used to standardize scores across all the sessions. The verbal working-memory index has a mean of zero, and each full point above or below zero represents $1 \mathrm{SD}$ of the standardized score. Positive values represent better memory function, and negative values poorer function. Fifteen patients could not provide valid data from at least one of the tasks because of the severity of psychosis or global impairment.

\section{SIDE EFFECTS AND ADVERSE EVENTS}

Movement disorders were assessed by the Extrapyramidal Symptom Rating Scale (ESRS) ${ }^{24}$ and the Barnes Akathisia Scale (BAS). ${ }^{25}$ The ESRS score ranges from 0 to 246, with subscales for parkinsonism (0 to 108), dystonia (0 to 96), and dyskinesia (0 to 42). The BAS score ranges from 0 to 5 . Higher scores on both scales are associated with more severe symptoms. General side effects were assessed according to a standardized, 42-item scale, the Udvalg for Kliniske Unders $\phi$ gelser $^{26}$ (range, 0 to 126), with higher scores indicating more severe side effects. Weight, waist circumference, and height were measured. Because clozapine can be associated with metabolic disturbances, fasting blood glucose, total cholesterol, low-density lipoprotein (LDL) cholesterol, high-density lipoprotein (HDL) cholesterol, and triglycerides were measured in serum on days 7, 63, and 189. White-cell counts were carried out weekly during the double-blind phase and according to local practice thereafter.

\section{STATISTICAL ANALYSIS}

Samples of 40 to 100 patients are recommended for studies of drug augmentation in schizophrenia, on the basis of expected effect sizes of 0.5 to 0.8. ${ }^{6,27-29}$ Effect sizes were determined with the use of Cohen's d statistic, which provides a measure of the differences in the mean values of 
changes in symptom severity between groups in relation to the pooled standard deviation. ${ }^{30} \mathrm{Ef}-$ fect sizes were categorized as small (0.2 to 0.4$)$, medium ( 0.5 to 0.7$)$, or large ( 0.8 or greater). For example, in a group of patients with treatmentresistant schizophrenia, switching from treatment with typical antipsychotic agents to optimal clozapine treatment was associated with a large effect size (0.8) for improvement of overall symptom severity and a medium effect size $(0.5)$ for improvement of specific positive and negative symptoms. ${ }^{31}$ The relative benefit of atypical as compared with typical antipsychotic agents for long-term memory dysfunction in schizophrenia was associated with a small effect size (0.2). ${ }^{32}$

In the present study, the primary analysis was performed according to the intention-to-treat principle. The primary outcome measure was the total PANSS score. A mixed-model analysis was used (SPSS, version 13), which included all available data at each time point. This analysis included fixed effects for group (risperidone and placebo) and time (days 7, 35, and 63) and an unstructured covariance structure. The statistic analyzed for

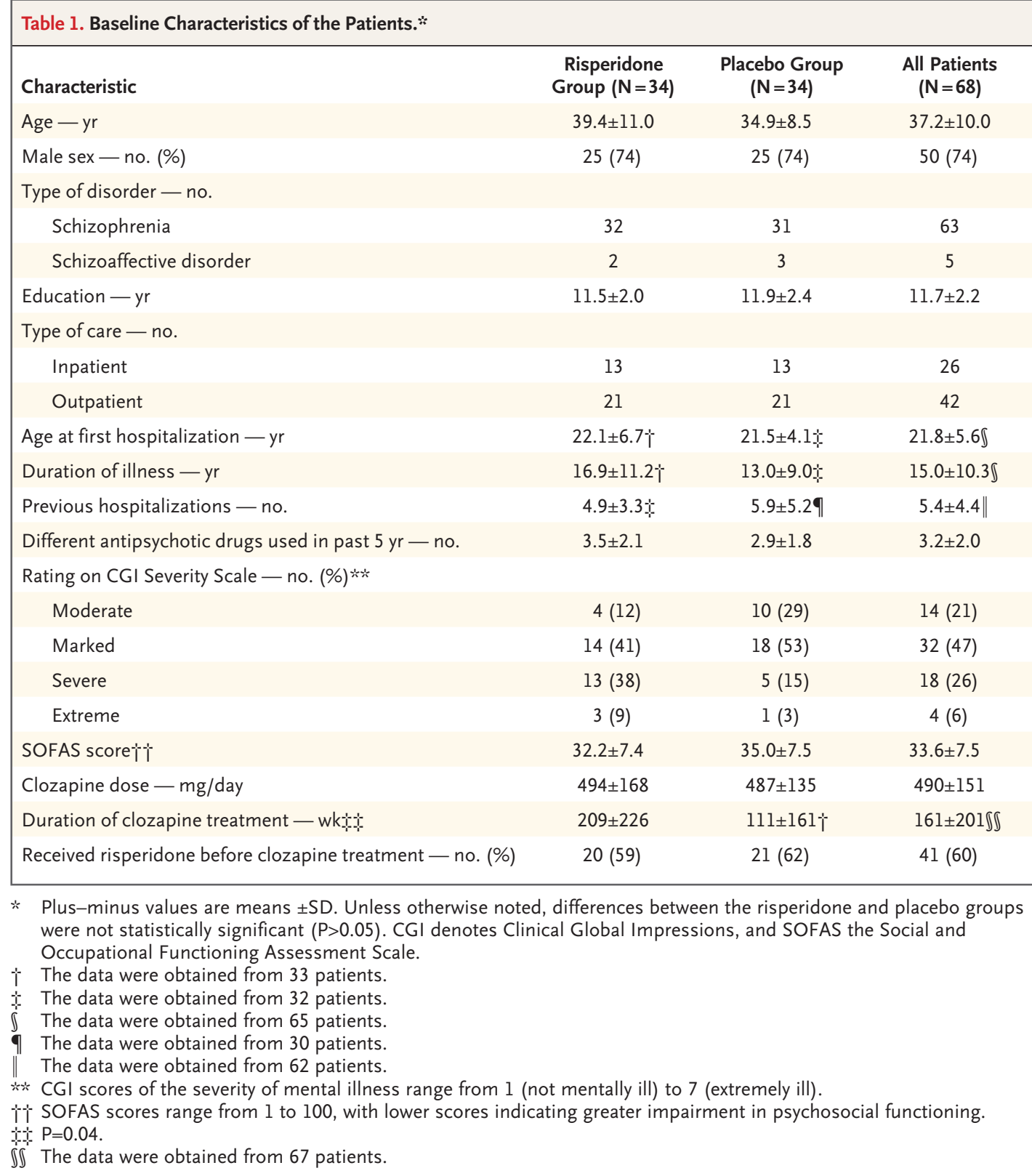


significance was the interaction term, which allows comparison of the treatment groups over time. For descriptive purposes, we also provide information on the differences between groups in the change from baseline to eight weeks and on effect sizes. In a secondary analysis, the proportions of patients having a response (those with a decrease in total PANSS score of at least 20 percent) in each group were compared by the chi-square test.

\section{RESULTS}

\section{STUDY POPULATION}

The investigators assessed 595 patients for eligibility for the study; 458 (77 percent) did not meet the inclusion criteria, and 69 (12 percent) declined to participate (see figure in the Supplementary Appendix, which is available with the full text of this article at www.nejm.org). A total of 71 patients were enrolled, 2 of whom withdrew consent before randomization. One patient improved during the first seven days of single-blind augmentation with placebo and no longer met the criteria for randomization. Seventy-two percent (49 patients) described themselves as white, 1 percent (1 patient) as black, 18 percent (12 patients) as
Asian, and 9 percent (6 patients) as belonging to another racial or ethnic group. The mean number of weeks of prior clozapine treatment was greater in the group randomized to risperidone $(\mathrm{P}=0.04)$. There were no other significant differences between the treatment groups in demographic or clinical characteristics (Table 1). There were no significant differences among the patients at the seven participating institutions in total PANSS score at baseline, age, sex ratio, educational level, age at first hospitalization, or number of previous hospitalizations.

\section{PRIMARY OUTCOME MEASURES}

The total PANSS score did not differ between the risperidone and placebo groups at baseline or at eight weeks of follow-up. A significant improvement between baseline and follow-up occurred in both groups $(\mathrm{P}<0.001)$. There was no difference between the risperidone and the placebo augmentation groups in the amount of improvement (Table 2 and Fig. 1). The mean difference in the change in PANSS scores from baseline to eight weeks between the two groups was 0.1 ( 95 percent confidence interval, -7.3 to 7.0 ). The effect sizes generated by these estimates would range from -0.50 to 0.49 . The change in the PANSS score

\begin{tabular}{|c|c|c|c|c|c|c|}
\hline \multirow[t]{2}{*}{ Outcome Measure } & \multicolumn{2}{|c|}{ Risperidone Group } & \multicolumn{2}{|c|}{ Placebo Group } & \multirow[t]{2}{*}{ P Value'̀ } & \multirow[t]{2}{*}{ Effect Size } \\
\hline & $\begin{array}{l}\text { Day } 7 \\
(\mathrm{~N}=34)\end{array}$ & $\begin{array}{l}\text { Day } 63 \\
(\mathrm{~N}=32)\end{array}$ & $\begin{array}{c}\text { Day } 7 \\
(\mathrm{~N}=34)\end{array}$ & $\begin{array}{l}\text { Day } 63 \\
(\mathrm{~N}=33)\end{array}$ & & \\
\hline \multicolumn{7}{|l|}{ PANSS score } \\
\hline Total & $102.5 \pm 14.6$ & $89.8 \pm 15.8$ & $97.8 \pm 12.4$ & $84.8 \pm 20.1$ & 0.96 & 0.01 \\
\hline Positive & $23.4 \pm 5.8$ & $20.4 \pm 5.7$ & $21.1 \pm 4.5$ & $18.4 \pm 5.4$ & 0.83 & 0.05 \\
\hline Negative & $27.8 \pm 5.5$ & $24.7 \pm 6.3$ & $27.3 \pm 6.3$ & $23.6 \pm 7.1$ & 0.24 & -0.09 \\
\hline CGI score for severity & $5.44 \pm 0.82$ & $5.03 \pm 0.97$ & $4.91 \pm 0.75$ & $4.52 \pm 1.06$ & 0.68 & 0.01 \\
\hline $\begin{array}{l}\text { Verbal working-memory } \\
\text { index』 }\end{array}$ & $0.09 \pm 0.83$ & $-0.08 \pm 0.99$ & $-0.10 \pm 0.85$ & $0.14 \pm 0.83$ & 0.02 & -0.68 \\
\hline
\end{tabular}

* Plus-minus values are means \pm SD. PANSS denotes the Positive and Negative Syndrome Scale, and CGI Clinical Global Impressions.

$\dagger \mathrm{P}$ values were calculated by the $\mathrm{F}$ test for the interaction between augmentation (risperidone or placebo) and time in the mixed-measures analysis.

$\ddagger$ The effect size was calculated by subtracting the mean score at day 63 from the score at day 7 for each group, determining the difference (risperidone minus placebo) between the two values, and then dividing this difference by the pooled standard deviation of the difference scores. Because improvement is indicated by lower scores for symptom severity but by higher scores on cognitive tests, a negative sign was added to the effect size of the verbal working-memory index for consistency. Negative signs indicate an advantage for placebo as compared with risperidone augmentation.

$\int$ The verbal working-memory index is a standardized composite $z$ score derived from the Letter-Number Sequencing and the Brown-Peterson tests. Sample sizes for the composite scores for the verbal working-memory index were 30 in the risperidone group and 23 in the placebo group at both day 7 and day 63. 
was not significantly correlated with the duration of previous clozapine treatment or with the duration of illness. When data for inpatients and outpatients were analyzed separately, the amount of improvement in the total PANSS score did not differ between inpatients (mean, 13.5) and outpatients (mean, 10.5) or between those receiving risperidone and those receiving placebo within either group.

\section{SECONDARY OUTCOME MEASURES}

The positive and negative symptom-severity scores showed a similar profile, as did the total scores, with significant improvement between baseline and eight weeks in both groups (positive $\mathrm{P}<0.001$, negative $\mathrm{P}<0.001$ ), but there were no significant differences between the risperidone and the placebo groups. There were no significant differences between the risperidone and the placebo groups in CGI severity or improvement score. Overall, 26 percent of the patients in the placebo augmentation group (9 of 34) were classified as having a response, as compared with 18 percent (6 of 34) in the risperidone augmentation group $(\mathrm{P}=0.38)$.

For verbal working memory, there was no difference between the two groups at baseline or at the eight-week follow-up in the composite score (Table 2). However, the amount of change between baseline and eight weeks differed significantly between the placebo group and the ris- peridone group $(\mathrm{P}=0.02)$; performance slightly increased in the placebo group and slightly declined in the risperidone group.

\section{DOSES}

At baseline and at the eight-week follow-up, the clozapine dose and the serum levels of clozapine and norclozapine did not differ between the risperidone and the placebo groups (Table 3). The mean dose of risperidone or the equivalent in placebo tablets did not differ between the two groups on days 35 and 63.

\section{SIDE EFFECTS AND ADVERSE EVENTS}

No statistically significant differences in the severity of movement disorders were observed between the placebo and risperidone groups (Table 3). Weight, waist circumference, and body-mass index did not differ significantly between the two groups at any time. For fasting blood glucose, there were no statistically significant differences between the two groups in the mean value at baseline or at the eight-week follow-up. However, fasting blood glucose increased more in the risperidone group than in the placebo group (16.2 vs. $1.8 \mathrm{mg}$ per deciliter [0.90 vs. $0.10 \mathrm{mmol}$ per liter], $\mathrm{P}=0.04$ for the comparison of the changes from baseline in the two groups). In the risperidone group, 6 of 25 patients with fasting blood glucose levels of less than $126 \mathrm{mg}$ per deciliter $(7.00$

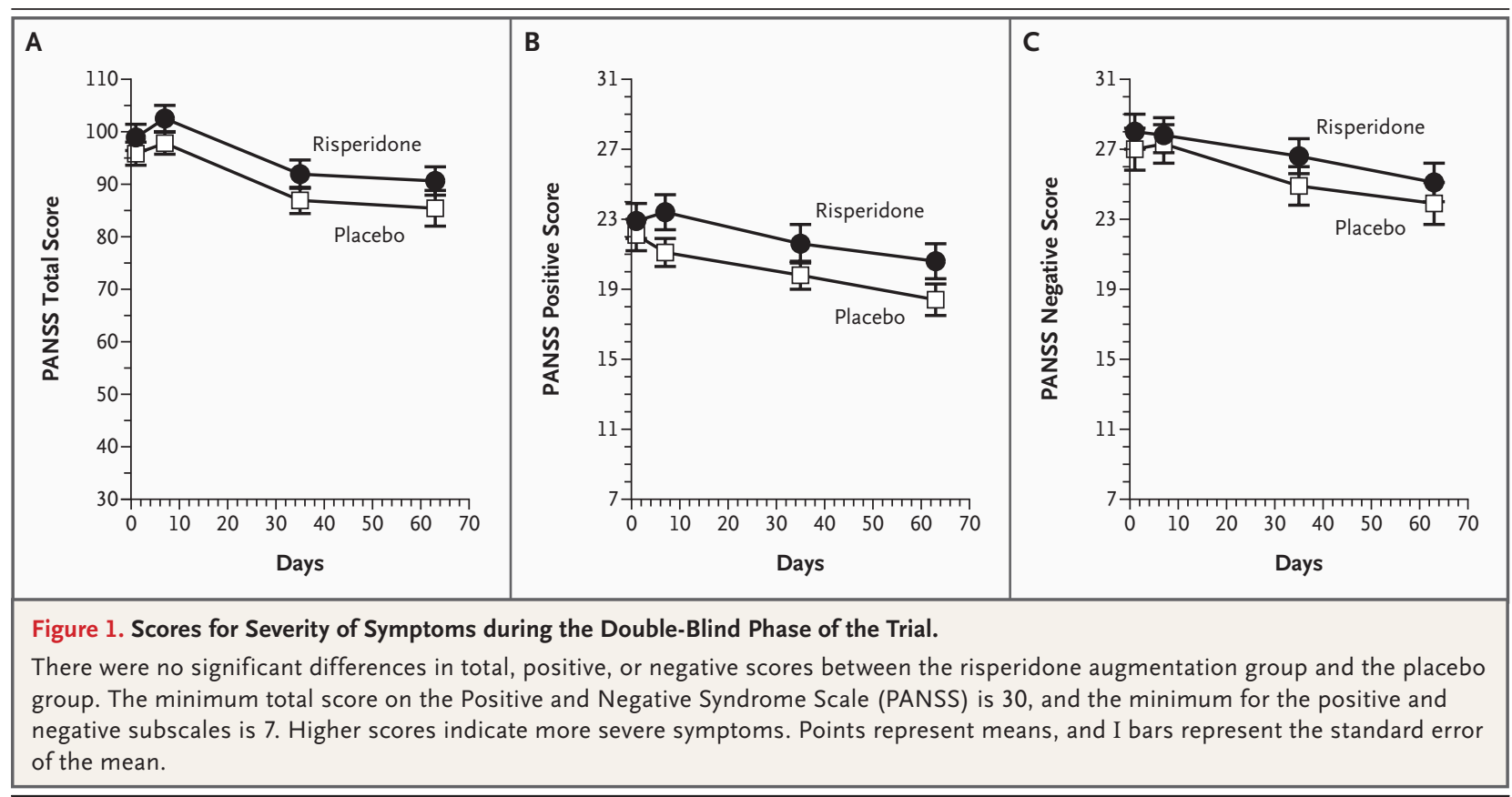




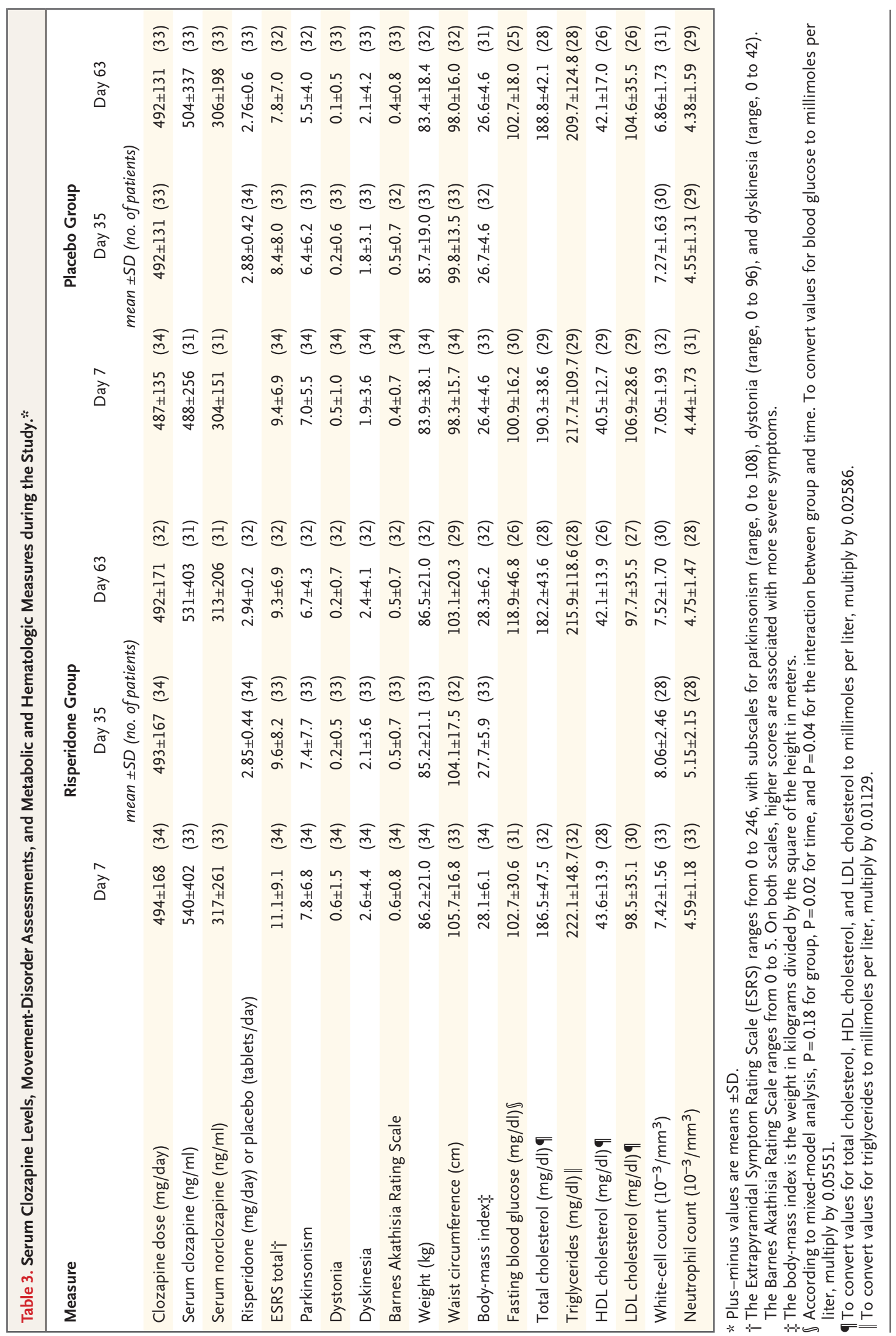


mmol per liter) at baseline had levels of $126 \mathrm{mg}$ per deciliter or more at eight weeks, as compared with 4 of 25 patients in the placebo group $(\mathrm{P}=0.73)$. There was no difference in the duration of previous treatment with clozapine between the patients whose blood glucose increased to at least $126 \mathrm{mg}$ per deciliter and those whose blood glucose did not. The levels of total cholesterol, triglycerides, LDL cholesterol, and HDL cholesterol did not differ significantly between the two groups at baseline or at eight weeks, and there were no significant differences between the two groups in the changes in these measurements between baseline and eight weeks. The total white-cell and neutrophil counts did not differ between the two groups. There were no statistically significant differences between the two groups in the incidence of any side effect (Table 4).
Three patients ( 4 percent) discontinued treatment before the eight-week follow-up. One patient each in the risperidone and placebo groups withdrew consent. The third patient, who was in the risperidone group, had a serious adverse event. His mental status deteriorated over one to two weeks, he required frequent restraint, and his creatine kinase level was elevated (with no evidence of fever, rigidity, or autonomic instability). He had a history of neuroleptic malignant syndrome related to haloperidol. Treatment with the study medication was stopped, and the patient was admitted to a medical ward. He subsequently recovered fully, from a medical perspective, and his mental status returned to the prestudy level of symptoms. Two other serious adverse events were reported, both during the extension phase of the study, when clozapine plus open-label risperidone

\begin{tabular}{|c|c|c|c|c|}
\hline \multirow[t]{3}{*}{ Effect } & $\begin{array}{l}\text { Placebo Group } \\
\qquad(\mathrm{N}=33)\end{array}$ & $\begin{array}{l}\text { Risperidone Group } \\
\qquad(\mathrm{N}=33)\end{array}$ & $\begin{array}{l}\text { Placebo Group } \\
\qquad(\mathrm{N}=32)\end{array}$ & $\begin{array}{c}\text { Risperidone Group } \\
(\mathrm{N}=32)\end{array}$ \\
\hline & \multicolumn{2}{|c|}{ Day 7} & \multicolumn{2}{|c|}{ Day 63} \\
\hline & \multicolumn{4}{|c|}{ no. with effect (\%) } \\
\hline Concentration difficulties & $19(58)$ & $18(55)$ & $11(34)$ & $21(66)$ \\
\hline $\begin{array}{l}\text { Asthenia, lassitude, or } \\
\text { increased fatigability }\end{array}$ & $17(52)$ & $18(55)$ & $13(41)$ & $14(44)$ \\
\hline Sleepiness or sedation & $13(39)$ & $19(58)$ & $13(41)$ & $19(59)$ \\
\hline Failing memory & $10(30)$ & $10(30)$ & $7(22)$ & $8(25)$ \\
\hline Depression & $11(33)$ & $8(24)$ & $10(31)$ & $12(38)$ \\
\hline Tension or inner unrest & $11(33)$ & $10(30)$ & $13(41)$ & $16(50)$ \\
\hline Increased duration of sleep & $10(30)$ & $12(36)$ & $11(34)$ & $11(34)$ \\
\hline Emotional indifference & $10(30)$ & $12(36)$ & $9(28)$ & $10(31)$ \\
\hline Increased salivation & $20(61)$ & $17(52)$ & $16(50)$ & $20(63)$ \\
\hline Constipation & $7(21)$ & $8(24)$ & $7(22)$ & $5(16)$ \\
\hline Orthostatic dizziness & $9(27)$ & $11(33)$ & $8(25)$ & $8(25)$ \\
\hline Palpitations or tachycardia & $8(24)$ & $4(12)$ & $4(13)$ & $4(13)$ \\
\hline Weight gain & $6(18)$ & $8(24)$ & $7(22)$ & $7(22)$ \\
\hline Amenorrhea† & $1(11)$ & $2(22)$ & 0 & $1(13)$ \\
\hline \multicolumn{5}{|l|}{ Global } \\
\hline Assessed by patient & $23(70)$ & $15(45)$ & $20(63)$ & $19(59)$ \\
\hline Assessed by physician & $25(76)$ & $20(61)$ & $21(66)$ & $24(75)$ \\
\hline
\end{tabular}

* The numbers and percentages of patients who had side effects scored as 1 (mild) or higher according to the Udvalg for Kliniske Undersøgelser on day 7 or day 63 are shown. According to this scale, each item is rated from 0 to 3, with higher scores indicating more severe side effects. Movement-disorder side effects are not shown, since these were assessed according to more detailed scales. Only side effects that occurred in at least 20 percent or more of the patients in a treatment group are reported. For all side effects, there were no statistically significant differences between the risperidone and placebo groups in the proportions of patients reporting the effect on day 7 or day 63 (all $P$ values $>0.05$ ). $\uparrow$ The data are based on nine women in each of the two groups on day 7 and eight women on day 63. 
was administered. One patient had an exacerbation of auditory hallucinations and suicidal ideation, requiring admission to the hospital. Poor adherence with treatment was suspected, and these symptoms had occurred before the patient entered the trial. A second patient had a self-inflicted scalp laceration that required stitches. There was a history of similar behavior involving head banging before the trial.

\section{EXTENSION PHASE}

A total of 46 patients completed the 18-week extension phase. At the end of the extension phase, the mean ( \pm SD) clozapine dose was $490 \pm 159 \mathrm{mg}$ per day and the mean risperidone dose was $2.8 \pm 0.8$ mg per day. We compared the improvement during the extension phase in patients who were randomized to placebo and those who were randomized to risperidone during the double-blind phase. There were no overall differences related to previous augmentation with placebo or risperidone. All patients continued to improve during the extension phase ( $\mathrm{P}=0.001$ for effect of time) (Fig. 2). There was no difference in improvement related to time between the two groups. The mean improvement in total PANSS score was 7.7 in the group that had previously received placebo augmentation and 5.0 in the group that had previously received risperidone augmentation. The pattern of results was similar for positive and negative symptoms.
The results from the extension phase also provide information about longer-term risperidone augmentation and cognition. Comparison of verbal working-memory function between day 7 and day 189 found no differences in the composite verbal working-memory score.

\section{DISCUSSION}

As compared with placebo, augmentation with risperidone in patients who had a limited response to clozapine alone offered no benefit during the eight-week, double-blind portion of this study. Our confidence intervals suggest that we can exclude a beneficial effect of risperidone augmentation that would be associated with a moderate-to-large effect size. In other words, if there is a beneficial effect of risperidone augmentation, the magnitude is likely to be smaller than the benefit associated with changing a group of treatment-resistant patients from typical antipsychotic drugs to clozapine. ${ }^{31}$ We may have failed to detect a smaller benefit or a benefit that might have emerged after a longer course of treatment. The present results are most consistent with those of a smaller study that reported an advantage of placebo over risperidone for augmentation of clozapine treatment. ${ }^{6}$ Both studies used a method of data analysis that included all time points sampled. Another study reported an advantage of risperidone augmentation ${ }^{7}$; this study analyzed only

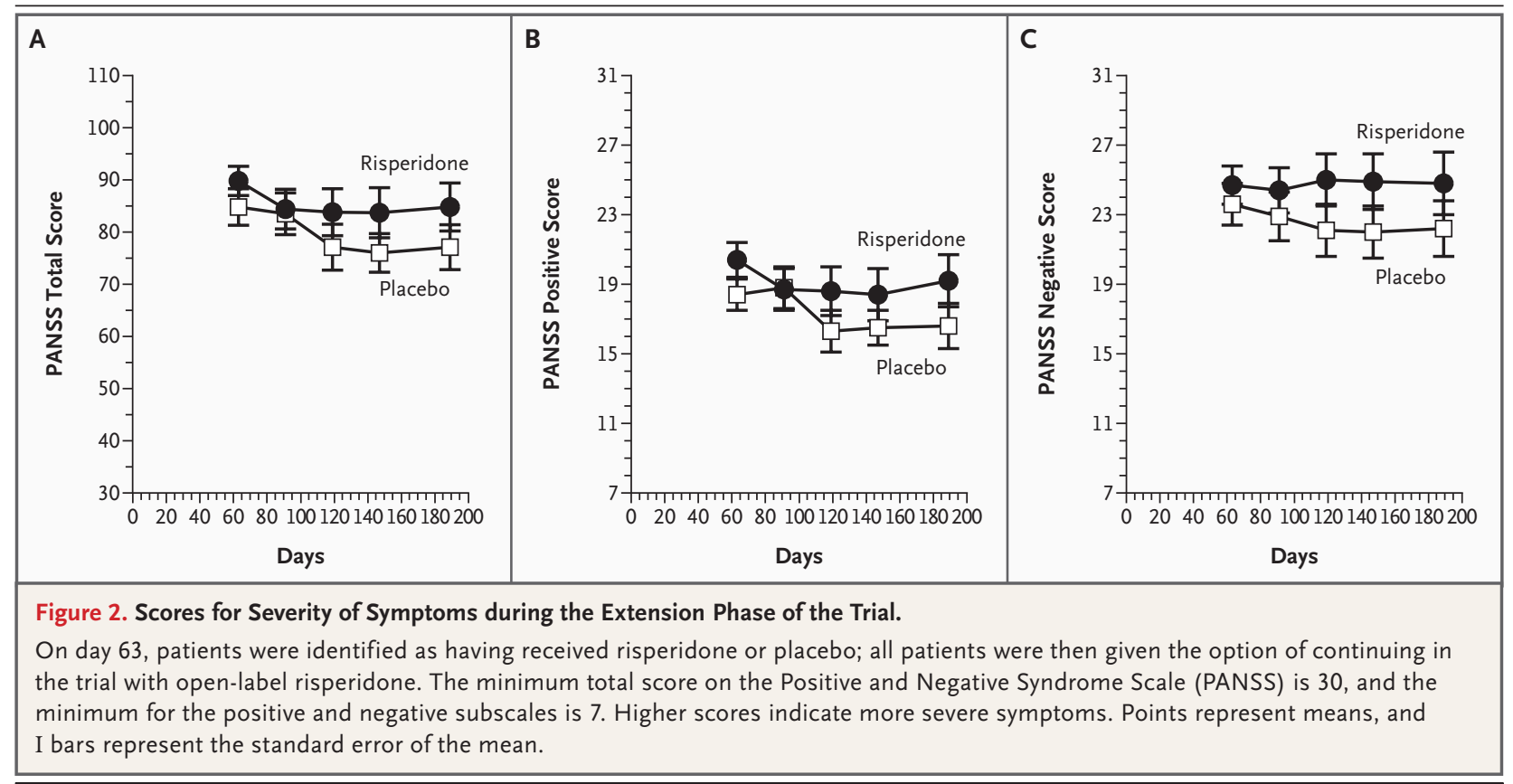


data taken at baseline and at the midpoint and end point of the study, even though the patients had been assessed more frequently. Smaller samples (40 patients or fewer) may have also accounted for inconsistent results in the earlier studies.

In each of these three controlled studies, all patients improved over time, particularly during weeks 2 to 6 . This improvement, which is associated with placebo augmentation in patients with chronic, refractory disease, is in contrast to the minimal effect of placebo alone observed in a meta-analysis of randomized clinical trials of atypical antipsychotic drugs for acute schizophrenia over a similar treatment period. ${ }^{33}$ The nonspecific effects of being in a treatment trial may be greater in patients with chronic, refractory forms of schizophrenia than in those who are either having acute exacerbations or are in earlier phases of the illness. Moreover, although it was statistically significant, the degree of improvement was moderate, with most patients remaining severely ill even after at least four months of augmentation of clozapine with risperidone.

Atypical antipsychotic drugs appear to improve cognitive function or, at least, not to result in impairment. ${ }^{17}$ For cognitive tasks involving frontal-lobe function, risperidone appears to have an advantage over clozapine in verbal working memory. ${ }^{17}$ In the present study, this aspect of frontallobe function was impaired in patients receiving augmentation with risperidone as compared with placebo. Other investigations of risperidone augmentation of clozapine have reported an increase in prolactin, which is probably related to increased occupancy of dopamine D2 receptors. ${ }^{34}$ Although the precise role of dopamine D2 receptors in cog- nitive function remains unclear, increased occupancy of these receptors may have adverse consequences. ${ }^{35,36}$

Impairment of glucose regulation may be more frequent with antipsychotic polypharmacy than with monotherapy. ${ }^{37}$ Clozapine monotherapy is associated with an increased risk of diabetes, ${ }^{38}$ and the present study suggests that risperidone augmentation may add to the risk of impaired glucose regulation. However, this finding is based on a subgroup of patients and must be regarded as preliminary.

In conclusion, our findings offer no support for antipsychotic polypharmacy, at least for the treatment of refractory forms of schizophrenia with a poor response to clozapine.

Supported by a grant from the Stanley Medical Research Institute. Risperidone and placebo were provided by JanssenOrtho, Canada.

Dr. Honer reports having received consulting or advisory board fees from In Silico Biosciences, Janssen, and Wyeth and lecture fees from AstraZeneca; Dr. Chen, grant funding from AstraZeneca; Dr. Falkai, consulting or advisory board fees and lecture fees from AstraZeneca, Janssen, Eli Lilly, Organon, and Pfizer; Dr. Stip, consulting or advisory board fees from AstraZeneca, Janssen, El Lilly, and Pfizer, lecture fees from AstraZeneca, Janssen, and Eli Lilly, and grant funding from AstraZeneca; Dr. Williams, consulting or advisory board fees from AstraZeneca, Genpharm, Janssen, Eli Lilly, and Prestwick Pharmaceuticals, lecture fees from AstraZeneca, Genpharm, Janssen, Eli Lilly, Novartis, Pfizer, and Prestwick Pharmaceuticals, and grant funding from AstraZeneca, Janssen, and Pfizer; Dr. MacEwan, consulting or advisory board fees from AstraZeneca, Janssen, Eli Lilly, and Novartis, lecture fees from GlaxoSmithKline, and grant support from AstraZeneca; Dr. Wasan, consulting or advisory board fees from ALZA, Biodelivery Sciences, and Forbes Medi-Tech, grant funding from Eisai Pharmaceuticals and Enzon Pharmaceuticals, and a collaborative grant from Novartis and the National Cancer Institute of Canada; and Dr. Procyshyn, consulting or advisory board fees and lecture fees from AstraZeneca, GlaxoSmithKline, Janssen, and Eli Lilly. No other potential conflict of interest relevant to this article was reported.

\section{APPENDIX}

The following institutions and investigators participated in the CARE Study Group: Castle Peak Hospital, Hong Kong, China: S.P. Leung and J.O.Y. Wong; Centre de Recherche Fernand-Seguin, Montreal: J.P. Rodriguez, P. Lalonde, and E. Stip: Fulbourn Hospital, Cambridge, United Kingdom: E. Pomarol-Clotet and P.J. McKenna; Peace Arch Hospital, White Rock, B.C., Canada: G.W. MacEwan; Queen Mary Hospital, Hong Kong, China: E.Y.H. Chen and R.C.K. Chan; Riverview Hospital, Port Coquitlam, B.C., Canada: S.W. Flynn, S. Altman, W.G. Honer, A.E. Thornton, R. Procyshyn, S. Huckin, T. Au, V. Boudreau, and B. Humphries; Royal Jubilee Hospital, Victoria, B.C., Canada: R. Williams; University of British Columbia, Vancouver, Canada: K. Wasan; University Clinic, Homburg, Germany: A. Bergmann, P. Falkai, T. Wobrock, and H. Wollny.

REFERENCES

1. Freedman R. Schizophrenia. N Engl J Med 2003;349:1738-49.

2. Tapp A, Wood AE, Secrest L, Erdmann

J, Cubberley L, Kilzieh N. Combination antipsychotic therapy in clinical practice. Psychiatr Serv 2003;54:55-9.

3. Sernyak MJ, Rosenheck R. Clinicians reasons for antipsychotic coprescribing. J Clin Psychiatry 2004;65:1597-600.

4. Procyshyn RM, Thompson B. Patterns of antipsychotic utilization in a tertiary care psychiatric institution. Pharmacopsychiatry 2004;37:12-7.

5. Paton C, Lelliott $P$, Harrington $M$ Okocha C, Sensky T, Duffett R. Patterns of antipsychotic and anticholinergic prescribing for hospital inpatients. J Psychopharmacol 2003;17:223-9.

6. Anil Yagcioglu AE, Kivircik Akdede $\mathrm{BB}$, Turgut TI, et al. A double-blind con- trolled study of adjunctive treatment with risperidone in schizophrenic patients partially responsive to clozapine: efficacy and safety. J Clin Psychiatry 2005;66:6372.

7. Josiassen RC, Joseph A, Kohegyi E, et al. Clozapine augmented with risperidone in the treatment of schizophrenia: a randomized, double-blind, placebo-controlled trial. Am J Psychiatry 2005;162:130-6. 
8. Faries $\mathrm{D}$, Ascher-Svanum $\mathrm{H}$, Zhu $\mathrm{B}$ Correll C, Kane J. Antipsychotic monotherapy and polypharmacy in the naturalistic treatment of schizophrenia with atypical antipsychotics. BMC Psychiatry 2005;5:26.

9. Stahl SM. Antipsychotic polypharmacy: evidence-based prescribing or prescribing-based evidence? Int J Neuropsychopharmacol 2004;7:113-6.

10. Centorrino F, Goren JL, Hennen J, Salvatore P, Kelleher JP, Baldessarini RJ. Multiple versus single antipsychotic agents for hospitalized psychiatric patients: case-control study of risks versus benefits. Am J Psychiatry 2004;161:700-6.

11. Waddington JL, Youssef HA, Kinsella A. Antipsychotic polypharmacy and ab sence of adjunctive anticholinergics over the course of a 10-year prospective study. Br J Psychiatry 1998;173:325-9.

12. Chakos M, Lieberman J, Hoffman E, Bradford D, Sheitman B. Effectiveness of second-generation antipsychotics in patients with treatment-resistant schizophrenia: a review and meta-analysis of randomized trials. Am J Psychiatry 2001, 158:518-26.

13. Rosenheck R, Cramer J, Xu W, et al A comparison of clozapine and haloperi dol in hospitalized patients with refractory schizophrenia. N Engl J Med 1997;337:809 15.

14. Kane J, Honigfeld G, Singer J, Meltzer $\mathrm{H}$. Clozapine for the treatment-resistant schizophrenic: a double-blind comparison with chlorpromazine. Arch Gen Psy chiatry 1988;45:789-96.

15. Freudenreich O, Goff DC. Antipsychotic combination therapy in schizo phrenia: a review of efficacy and risks of current combinations. Acta Psychiatr Scand 2002;106:323-30.

16. Kapur S, Zipursky RB, Remington G. Clinical and theoretical implications of 5-HT2 and D2 receptor occupancy of clozapine, risperidone, and olanzapine in schizophrenia. Am J Psychiatry 1999;156: 286-93.

17. Woodward ND, Purdon SE, Meltzer
HY, Zald DH. A meta-analysis of neuropsychological change to clozapine, olanzapine, quetiapine, and risperidone in schizophrenia. Int J Neuropsychopharmacol 2005;8:457-72.

18. Diagnostic and statistical manual of mental disorders, 4th ed.: DSMV-IV. Washington, D.C.: American Psychiatric Association, 1994

19. Kay SR, Opler LA, Lindenmayer JP. Reliability and validity of the Positive and Negative Syndrome Scale for schizo phrenics. Psychiatry Res 1988;23:99-110.

20. Guy W. ECDEU assessment manua for psychopharmacology, revised. Rockville, Md.: Department of Health, Education, and Welfare, 1976. (Document no. ADM 76-338.)

21. Peterson LR, Peterson MJ. Short-term retention of individual verbal items. J Exp Psychol 1959;58:193-8.

22. Brown J. Some tests of the decay the ory of immediate memory. Q J Exp Psychol 1958;10:12-21.

23. The WAIS-III-WMS-III technica manual. San Antonio, Tex.: The Psycho logical Corporation, 1997.

24. Chouinard G, Ross-Chouinard A, Annable L, Jones BD. The Extrapyramida Symptom Rating Scale. Can J Neurol Sci 1980;7:233.

25. Barnes TRE. A rating scale for drug induced akathisia. Br J Psychiatry 1989, 154:672-6.

26. Lingjaerde O, Ahlfors UG, Bech P, Dencker SJ, Elgen K. The UKU side effect rating scale: a new comprehensive rating scale for psychotropic drugs and crosssectional study of side effects in neuroleptic-treated patients. Acta Psychiatr Scand Suppl 1987;334:1-100.

27. Stern RG, Schmeidler J, Davidson M. Limitations of controlled augmentation trials in schizophrenia. Biol Psychiatry 1997;42:138-43.

28. Taylor CG, Flynn SW, Altman S, Ehm ann T, MacEwan GW, Honer WG. An open trial of risperidone augmentation of partial response to clozapine. Schizophr Res 2001;48:155-8.
29. Henderson DC, Goff DC. Risperidone as an adjunct to clozapine therapy in chronic schizophrenics. J Clin Psychiatry 1996;57:395-7.

30. Cohen J. Statistical power analysis for the behavioral sciences. 2nd ed. Hillsdale, N.J.: Lawrence Erlbaum Associates, 1988. 31. Pickar D, Bartko JJ. Effect size of symptom status in withdrawal of typical antipsychotics and subsequent clozapine treatment in patients with treatmentresistant schizophrenia. Am J Psychiatry 2003;160:1133-8

32. Thornton AE, Van Snellenberg JX, Sepehry AA, Honer W. The impact of atypical antipsychotic medications on long-term memory dysfunction in schizophrenia spectrum disorder: a quantitative review. J Psychopharmacol (in press).

33. Sherwood M, Thornton AE, Honer WG. A meta-analysis of profile and timecourse of symptom change in acute schizophrenia treated with atypical antipsychotics. Int J Neuropsychopharmacol 2005;9:1-10.

34. Henderson DC, Goff DC, Connolly CE, Borba CP, Hayden D. Risperidone added to clozapine: impact on serum prolactin levels. J Clin Psychiatry 2001;62:6058.

35. Reeves SJ, Grasby PM, Howard RJ, Bantick RA, Asselin M-C, Mehta MA. A positron emission tomography (PET) investigation of the role of striatal dopamine (D2) receptor availability in spatial cognition. Neuroimage 2005;28:216-26. 36. Wang M, Vijayraghavan S, GoldmanRakic PS. Selective D2 receptor actions on the functional circuitry of working memory. Science 2004;303:853-6.

37. Taylor D, Young C, Esop R, Paton C, Walwyn R. Testing for diabetes in hospitalised patients prescribed antipsychotic drugs. Br J Psychiatry 2004;185:152-6. 38. Newcomer JW. Abnormalities of glucose metabolism associated with atypical antipsychotic drugs. J Clin Psychiatry 2004;65:Suppl 18:36-46.

Copyright (c) 2006 Massachusetts Medical Society. 